\title{
Recommendations on SARS-CoV-2 vaccination in adult patients with rheumatic diseases
}

Min Shen ${ }^{1}$, Lingli Dong², Mengtao Li ${ }^{1}$, Yan Zhao ${ }^{1 *}$, Xiaofeng Zeng ${ }^{1 *}$, on behalf of the Chinese Rheumatology Association\#

${ }^{1}$ Department of Rheumatology and Clinical Immunology, Chinese Academy of Medical Sciences \& Peking Union Medical College; National Clinical Research Center for Dermatologic and Immunologic Diseases (NCRC-DID), Ministry of Science \& Technology, State Key Laboratory of Complex Severe and Rare Diseases, Peking Union Medical College Hospital (PUMCH), Key Laboratory of Rheumatology and Clinical Immunology, Ministry of Education, Beijing, China

${ }^{2}$ Department of Rheumatology and Immunology, Tongji Hospital, Tongji Medical College, Huazhong University of Science and Technology, Wuhan, China

Received November 10, 2021 accepted November 11, 2021

Abstract

Vaccination against coronavirus disease 2019 (COVID-19) has been promoted all over the world and has become an important measure to control the pandemic. Patients with rheumatic diseases are at high risk of 2019-nCoV severe infection, hence are the target population with high priority for vaccination. In 2021, under the leadership of the Chinese Rheumatology Association, the recommendations on SARS-CoV-2 vaccination for adult patients with rheumatic diseases in China were proposed based on the current data, in combination with international guidelines and experts' opinions.

Keywords

SARS-CoV-2・vaccine $\cdot$ rheumatic diseases $\bullet$ guideline

\section{Overarching Recommendations}

- Individual and social factors should be fully considered when making the decision of SARS-CoV-2 vaccination for adult patients with rheumatic diseases. The decision-making process should be jointly implemented by rheumatologists, the vaccination physician, the primary care physician and the patient.

- SARS-CoV-2 vaccination in adult patients with rheumatic diseases should preferably be administered during quiescent disease if there is no other contraindication.

- The use of immunosuppressants may reduce the effectiveness of the vaccine.

- The majority of immunosuppressive agents, biological agents and targeted synthetic DMARDs (disease modify anti-rheumatic drugs) should be used continuously with no modification on the time of vaccination, except for methotrexate, JAK inhibitors, abatacept, cyclophosphamide, and rituximab, for which optimizing the timing of immunosuppressive therapy and vaccination is recommended.

\section{Characteristics of adult patients with rheumatic diseases}

Due to the dysfunction of immune systems, patients with rheumatic diseases are at higher risk of 2019-nCoV infections with worse clinical outcomes in contrast to individuals without rheumatic diseases. ${ }^{[1,2]}$ Thus, these patients should be prioritized to receive SARS-CoV-2 vaccination compared with the general population with similar age and gender.

The following recommendations are for adult (over 18 years old) patients with rheumatic diseases (over 18 years old) 
who fulfill the conditions listed in the "Technical Guidelines of SARS-CoV-2 vaccination (First Edition, China)". In this recommendation, rheumatic diseases are termed as autoimmune diseases, inflammatory rheumatic diseases and autoinflammatory diseases, including but not limited to rheumatoid arthritis, spondyloarthropathy, systemic lupus erythematosus, Sjogren's syndrome, myositis/dermatomyositis, systemic sclerosis, mixed connective tissue disease, antiphospholipid syndrome, systemic vasculitis, IgG4-related diseases, relapsing polychondritis, polymyalgia rheumatica, adult-onset Still's disease, familial Mediterranean fever, and so on.

\section{Recommendations for SARS-CoV-2 vaccination}

The following recommendations were summarized based on the current data and "Technical Guidelines of SARS-CoV-2 vaccination (First Edition, China)", combined with the available evidence derived from other vaccination in patients with rheumatic diseases, ${ }^{[3]}$ the guidance for COVID-19 vaccination in patients with rheumatic and musculoskeletal diseases published by American College of Rheumatology (ACR) in March 2021 and the viewpoints on SARS-CoV-2 vaccination in patients with rheumatic and musculoskeletal diseases published by The European League Against Rheumatism (EULAR) in February 2021. ${ }^{[4]}$

\section{Vaccine types}

Administration of inactivated vaccine is the first choice for adult patients with rheumatic diseases, whereas other types of vaccines, such as recombinant subunit vaccine, adenovirus vector vaccine, mRNA vaccine, should be considered with caution.

\section{Indications}

Adult patients with quiescent disease can be vaccinated except for those with contraindications listed below. Vaccination during the active disease needs further investigation. However, the effectiveness of vaccine in rheumatic patients under immunosuppressive therapy might be decreased than expected when compared to that in people without rheumatic diseases.

\section{Contraindications}

Patients with contraindications mentioned in the "Technical Guidelines of SARS-CoV-2 vaccination (First Edition, China)" cannot be vaccinated.

\section{Modification of immunosuppressive therapy and vaccination} The majority of immunosuppressive agents, biological agents and targeted synthetic DMARDs should be used continuously with no modification on the time of vaccination. However, optimizing the vaccination time is recommended for patients treated with methotrexate, JAK inhibitors, abatacept, cyclophosphamide (intravenous) and rituximab (Table 1).
Table 1. Modifications of immunosuppressive therapy and SARSCoV-2 vaccination in adult patients with rheumatic diseases

\begin{tabular}{|c|c|}
\hline Drugs & $\begin{array}{l}\text { Immunosuppressive } \\
\text { therapy modifications and } \\
\text { SARS-CoV-2 vaccination } \\
\text { time }\end{array}$ \\
\hline $\begin{array}{l}\text { Glucocorticoids (prednisone equivalent dose } \\
<20 \text { mg/day) } \\
\text { Hydroxychloroquine } \\
\text { Leflunomide } \\
\text { Sulfasalazine } \\
\text { Mycophenolate mofetil } \\
\text { Azathioprine } \\
\text { Cyclophosphamide (oral) } \\
\text { Oral calcineurin inhibitors (such as ciclosporin } \\
\text { and tacrolimus) } \\
\text { TNF inhibitors (such as certolizumab, etaner- } \\
\text { cept, adalimumab, infliximab, golimumab) } \\
\text { IL-6 antagonists (such as tocilizumab, sari- } \\
\text { lumab) } \\
\text { IL-1 antagonist (such as anakinra, canakinum- } \\
\text { ab) } \\
\text { IL-17 inhibitors (such as secukinumab, ixeki- } \\
\text { zumab) } \\
\text { IL-12/ IL-13 antagonist (such as ustekinumab) } \\
\text { IL-23 antagonist (such as rizankizumab, } \\
\text { guselkumab) } \\
\text { Belimumab }\end{array}$ & No modification \\
\hline Methotrexate & $\begin{array}{l}\text { Withhold methotrexate } 1 \\
\text { week after each dose of } \\
\text { vaccine. No modification of } \\
\text { vaccination. }\end{array}$ \\
\hline $\begin{array}{l}\text { JAK inhibitors (such as tofacitinib, baricitinib, } \\
\text { upadacitinib) }\end{array}$ & $\begin{array}{l}\text { Withhold JAK inhibitors } 1 \\
\text { week after each dose of } \\
\text { vaccine. No modification of } \\
\text { vaccination. }\end{array}$ \\
\hline Abatacept (subcutaneous) & $\begin{array}{l}\text { Withhold abatacept both } \\
1 \text { week prior to and } 1 \text { week } \\
\text { after the first dose of vac- } \\
\text { cine. No interruption for the } \\
\text { second dose of vaccine. }\end{array}$ \\
\hline Cyclophosphamide (intravenous) & $\begin{array}{l}\text { Administrating the cyclo- } \\
\text { phosphamide } 1 \text { week after } \\
\text { each dose of vaccine. }\end{array}$ \\
\hline Rituximab & $\begin{array}{l}\text { Preferably vaccinate prior to } \\
\text { the initiation of rituximab. } \\
\text { For patients using rituximab } \\
\text { because of the disease } \\
\text { activity, vaccination should } \\
\text { be provided at the follow- } \\
\text { ing time window: at least } \\
6 \text { months after rituximab } \\
\text { administration, and at least } \\
4 \text { weeks prior to the next } \\
\text { administration of rituximab; } \\
\text { delaying the administration } \\
\text { of rituximab for } 2-4 \text { weeks } \\
\text { after the second dose of } \\
\text { vaccine if possible. } \\
\text { The timing could be applied } \\
\text { to the patients whose } \\
\text { conditions are allowed for } \\
\text { a long period of rituximab } \\
\text { withdrawal and it might not } \\
\text { be suitable for all patients. }\end{array}$ \\
\hline
\end{tabular}

TNF, tumor necrosis factor; IL, interleukin; JAK, Janus kinase.

\section{Special attentions}

Rheumatologists should be involved in the assessment of indications for SARS-CoV-2 vaccination in adult patients with 
rheumatic diseases. Individualized schedule for vaccination should be explained to the patient by the rheumatologist and be jointly implemented by rheumatologists, the vaccination physician, the primary care physician and the patient.

Theoretically, there might be a risk of flares or progression of underlying diseases after SARS-CoV-2 vaccination, yet the expected benefits of vaccination in patients with rheumatic diseases could overweight the potential risks of flares of preexisting disease. Nevertheless, disease activity should be closely monitored after vaccination.

Since March 2021, vaccine-related venous thrombosis, especially thrombosis at rare locations (for example, intracranial venous sinus thrombosis) following AstraZeneca COVID-19 vaccination (adenovirus vector vaccines), were reported in Europe, which is termed vaccine-induced prothrombotic immune thrombocytopenia (VIPIT). ${ }^{[5]}$ It is recommended that patients with a history of thrombosis and/or known thrombophilia, such as antiphospholipid syndrome, should avoid the adenovirus vector vaccines, although there is no evidence showing higher risk for complications of thrombosis in intracranial veins or other rare sites after AstraZeneca COVID-19 vaccination.

Other public health guidelines, such as social distance and individual preventive measures, should be followed in patients with rheumatic diseases even after being vaccinated.

Family members and other close contacts of patients with rheumatic diseases should be vaccinated against COVID-19. This might benefit the patients.

For those patients with rheumatic diseases who recovered from the COVID-19 infections or will being vaccinated with other vaccines simultaneously, please refer to the "Technical Guidelines of SARS-CoV-2 vaccination (First Edition, China)".

Conflict of Interest

Xiaofeng Zeng is the Editor-in-Chief of the journal, and Mengtao Li and Yan Zhao are Associate Editors-in-Chief. The article was subject to the journal's standard procedures, with peer review handled independently of these members and their research groups.

\section{Expert Panel}

Lie Dai, Department of Rheumatology, Sun Yat-Sen Memorial Hospital, Sun Yat-Sen University Lan He, Department of Rheumatology and Immunology, The First Affiliated Hospital of Xi'an Jiaotong University Cibo Huang, Department of Rheumatology and Clinical Immunology, South China Hospital of Shenzhen University Caifeng Li, Department of Rheumatology, Beijing Children's Hospital, Capital Medical University, National Center for Children's Health Xiaomei Li, Department of Rheumatology and Immunology, The First Affiliated Hospital of USTC, Division of Life Sciences and Medicine, University of Science and Technology of China

Jin Lin, Department of Rheumatology and Immunology, The First Affiliated Hospital, Zhejiang University, School of Medicine Shengyun Liu, Department of Rheumatology and Immunology, The First Affiliated Hospital of Zhengzhou University Yi Liu, Department of Rheumatology and Immunology, West China Hospital, Sichuan University

Yin Su, Department of Rheumatology and Immunology, Peking University People's Hospital

Wei Wei, Department of Rheumatology and Immunology, Tianjin Medical University General Hospital

Lijun Wu, Department of Rheumatology and Immunology, People's Hospital of Xinjiang Uygur Autonomous Region

Huji Xu, Department of Rheumatology and Immunology, Changzheng Hospital, Naval Medical University

Chengde Yang, Department of Rheumatology and Immunology, Ruijin Hospital, Shanghai Jiao Tong University School of Medicine Niansheng Yang, Department of Rheumatology, The First Affiliated Hospital, Sun Yat-sen University

Pingting Yang, Department of Rheumatology and Immunology, The First Affiliated Hospital of China Medical University Liyun Zhang, Department of Rheumatology and Clinical Immunology, Shanxi Bethune Hospital, Shanxi Academy of Medical Sciences, Third Hospital of Shanxi Medical University

Xuan Zhang, Department of Rheumatology, Beijing Hospital, National Center of Gerontology, Institute of Geriatric Medicine, Chinese Academy of Medical Sciences

Xuewu Zhang, Department of Rheumatology and Immunology, Peking University People's Hospital

Zhiyi Zhang, Department of Rheumatology and Immunology, The First Affiliated Hospital of Harbin Medical University

Zhuoli Zhang, Department of Rheumatology and Clinical Immunology, Peking University First Hospital

Dongbao Zhao, Department of Rheumatology and Immunology, The First Affiliated Hospital of Naval Medical University

Xiaoxia Zuo, Department of Rheumatology and Immunology, Xiangya Hospital of Central South University

Dongbao Zhao, Department of Rheumatology and Clinical Immunology, the First Affiliated Hospital of Naval Military Medical University (Shanghai Changhai Hospital)

Xiaoxia Zuo, Department of Rheumatology and Clinical Immunology, Xiangya Hospital of Central South University 


\section{References}

[1] Akiyama S, Hamdeh S, Micic D, et al. Prevalence and clinical outcomes of COVID-19 in patients with autoimmune diseases: a systematic review and meta-analysis. Ann Rheum Dis. 2021;80(3):384391.

[2] Curtis JR, Johnson SR, Anthony DD, et al. American College of Rheumatology Guidance for COVID-19 Vaccination in Patients with Rheumatic and Musculoskeletal Diseases - Version 1. Arthritis Rheumatology (Hoboken, NJ). 2021;73(10):e60-e75.

[3] Furer V, Rondaan C, Heijstek MW, et al. 2019 update of EULAR recommendations for vaccination in adult patients with autoimmune inflammatory rheumatic diseases. Ann Rheum Dis. 2020;79(1):39-52. [4] Bijlsma JW. EULAR December 2020 View points on SARS-CoV-2 vaccination in patients with RMDs. Ann Rheum Dis. 2021;80(4):411412.

[5] Oldenburg J, Klamroth R, Langer F, et al. Diagnosis and Management of Vaccine-Related Thrombosis following AstraZeneca COVID-19 Vaccination: Guidance Statement from the GTH. Hamostaseologie 2021. 